\title{
Primary care
}

\section{Cluster randomised controlled trial to compare three methods of promoting secondary prevention of coronary heart disease in primary care}

\author{
Michael Moher, Patricia Yudkin, Lucy Wright, Rebecca Turner, Alice Fuller, Theo Schofield, \\ David Mant for the Assessment of Implementation Strategies (ASSIST) Trial Collaborative Group
}

\begin{abstract}
Objective To assess the effectiveness of three different methods of promoting secondary prevention of coronary heart disease in primary care.

Design Pragmatic, unblinded, cluster randomised controlled trial.

Setting Warwickshire.

Subjects 21 general practices received intervention; outcome measured in 1906 patients aged 55-75 years with established coronary heart disease.

Interventions Audit of notes with summary feedback to primary health care team (audit group); assistance with setting up a disease register and systematic recall of patients to general practitioner (GP recall group); assistance with setting up a disease register and systematic recall of patients to a nurse led clinic (nurse recall group).

Main outcome measures At 18 months' follow up: adequate assessment (defined) of 3 risk factors (blood pressure, cholesterol, and smoking status); prescribing of hypotensive agents, lipid lowering drugs, and antiplatelet drugs; blood pressure, serum cholesterol level, and plasma cotinine levels.

Results Adequate assessment of all 3 risk factors was much more common in the nurse and GP recall groups $(85 \%, 76 \%)$ than the audit group $(52 \%)$. The advantage in the nurse recall compared with the audit group was 33\% (95\% confidence interval 19\% to $46 \%$ ); in the GP recall group compared with the audit group $23 \%$ (10\% to $36 \%)$, and in the nurse recall group compared with the GP recall group 9\% (-3\% to $22 \%$ ). However, these differences in assessment were not reflected in clinical outcomes. Mean blood pressure (148/80, 147/81, 148/81 $\mathrm{mm} \mathrm{Hg}$ ), total cholesterol $(5.4,5.5,5.5 \mathrm{mmol} / \mathrm{l})$, and cotinine levels (\% probable smokers 17\%, 16\%, 19\%) varied little between the nurse recall, GP recall, and audit groups respectively, as did prescribing of hypotensive and lipid lowering agents. Prescribing of antiplatelet drugs was higher in the nurse recall group (85\%) than the GP recall or audit groups $(80 \%, 74 \%)$. After adjustment for baseline levels, the advantage in the nurse recall group compared with the audit group was $10 \%$ (3\% to $17 \%)$, in the nurse recall group
\end{abstract}

compared with the GP recall group $8 \%$ (1\% to $15 \%)$ and in the GP recall group compared with the audit group $2 \%$ ( $-6 \%$ to $10 \%)$.

Conclusions Setting up a register and recall system improved patient assessment at 18 months' follow up but was not consistently better than audit alone in improving treatment or risk factor levels.

Understanding the reasons for this is the key next step in improving the quality of care of patients with coronary heart disease.

\section{Introduction}

Patients with established coronary heart disease are at serious risk of subsequent vascular events (non-fatal myocardial infarction, non-fatal strokes, and vascular deaths). ${ }^{1}$ This risk can be reduced by effective clinical and preventive care. ${ }^{2}$ Evidence also exists that the quality of such care in hospitals and general practices is inadequate. Assessment of risk is often incomplete, and many patients whose risk could be reduced are not receiving optimal treatment. ${ }^{34}$

Last year the national service framework for coronary heart disease set as a target in England that general practitioners and primary care teams should aim to identify all people with established cardiovascular disease and offer them comprehensive advice and appropriate treatment to reduce their risks. ${ }^{5}$ This will require important changes in clinical practice and in the systems of care. Methods of achieving quality improvement and change in clinical practice vary in their effectiveness, and these have recently been reviewed. ${ }^{6}$ Audit and feedback, the provision of guidelines (and facilitation to assist their adoption), record systems, improved communications between primary and secondary care, patient reminders, and nurse led clinics in general practice have all been advocated and tested as methods of producing change in practice for patients with established coronary heart disease..$^{7-12}$ They have not been compared directly and have different potential costs.

We aimed to compare the effectiveness of three different interventions for improving the secondary preventive care of patients with coronary heart disease delivered at the level of general practice: audit and

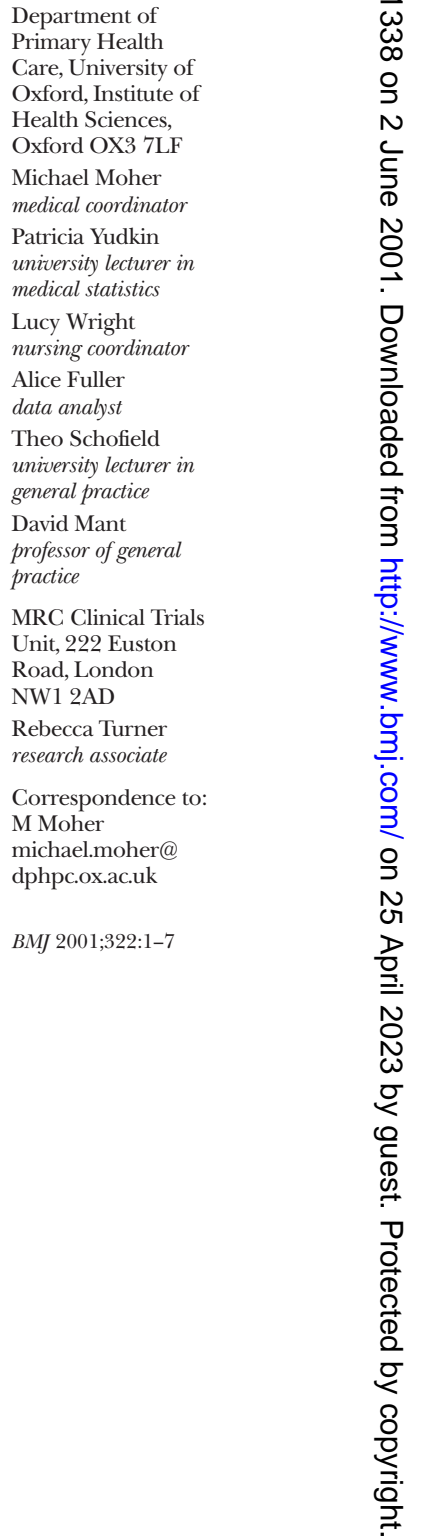




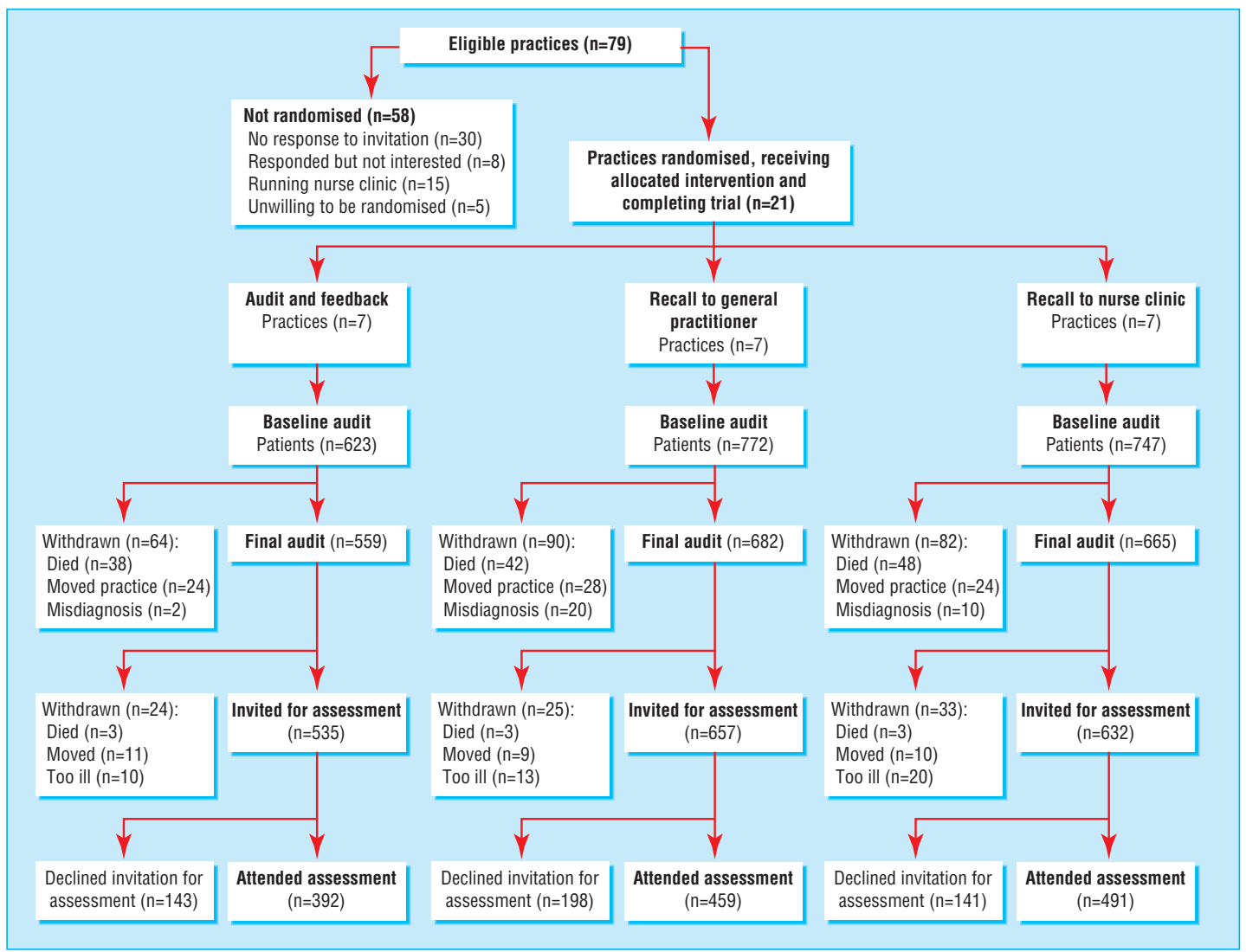

Flow of practices and patients through trial

feedback; recall to a general practitioner; and recall to a nurse clinic. The intervention was assessed in a pragmatic, unblinded, cluster randomised controlled trial that attempted to include all general practices in Warwickshire.

Table 1 Characteristics at baseline of all 79 general practices in Warwickshire, by whether interest was expressed in joining the study and whether they were recruited. Values are numbers (percentages) of practices unless stated otherwise

\begin{tabular}{|c|c|c|c|c|}
\hline & $\begin{array}{c}\text { Recruited } \\
(n=21)\end{array}$ & $\begin{array}{c}\text { Expressed } \\
\text { interest, not } \\
\text { recruited }(n=20)\end{array}$ & $\begin{array}{l}\text { Did not express } \\
\text { interest }(n=38)\end{array}$ & $\begin{array}{c}\text { P value for } \\
\text { difference } \\
\text { between groups }\end{array}$ \\
\hline \multicolumn{5}{|l|}{ List size: } \\
\hline$<6000$ & $7(33)$ & $5(25)$ & $25(66)$ & \multirow[t]{3}{*}{0.006} \\
\hline $6000-11999$ & $11(52)$ & $10(50)$ & $12(32)$ & \\
\hline$\geqslant 12000$ & $3(14)$ & $5(25)$ & $1(3)$ & \\
\hline \multicolumn{5}{|c|}{ General practitioners (whole time equivalent): } \\
\hline$<4$ & $10(48)$ & $5(25)$ & $27(71)$ & \multirow[t]{3}{*}{0.010} \\
\hline 4- & $7(33)$ & $11(55)$ & $9(24)$ & \\
\hline$\geqslant 6$ & $4(19)$ & $4(20)$ & $2(5)$ & \\
\hline \multicolumn{5}{|c|}{ Practice nurses (whole time equivalent): } \\
\hline$<1$ & $3(14)$ & $4(20)$ & $23(61)$ & \multirow[t]{3}{*}{$<0.001$} \\
\hline $1-$ & $8(38)$ & $5(25)$ & $10(26)$ & \\
\hline$\geqslant 2$ & $10(48)$ & $11(55)$ & $5(13)$ & \\
\hline Training practice & $7(33)$ & $11(55)$ & $8(21)$ & 0.040 \\
\hline $\begin{array}{c}\text { Computerised } \\
\text { practice }^{\star}\end{array}$ & $20(95)$ & $20(100)$ & $35(92)$ & 0.92 \\
\hline \multicolumn{5}{|l|}{ Location: } \\
\hline Rural & $5(24)$ & $2(10)$ & $8(21)$ & \multirow[t]{2}{*}{0.60} \\
\hline Urban & $16(76)$ & $18(90)$ & $30(79)$ & \\
\hline
\end{tabular}

*Practice that used a computer for clinical activities-for example, repeat prescribing and registration of patients.

\section{Participants and methods}

\section{Recruitment of practices}

In June 1997 we invited, by letter, all 79 general practices in Warwickshire to participate in the study; non-respondents to two letters were telephoned. The trial's medical coordinator (MM) visited or telephoned a nominated doctor in each practice to explain the purpose of the trial. Of the 41 practices that expressed interest, 20 withdrew (15 were ineligible because they were already running nurse led clinics and 5 were committed to opportunistic care). The remaining 21 practices were recruited (figure). The practices expressing no interest in the study were smaller, less likely to employ practice nurses, and less likely to be involved in training than others (table 1).

During the study the Warwickshire Medical Advisory Audit Group conducted an audit of the care of patients with coronary heart disease. Practices had to report audit results to qualify for health promotion payments.

\section{Identification of patients}

All paper and computer records of patients aged 55-75 years were hand searched by six external auditors, and patients with established coronary heart disease were identified. ${ }^{13}$ Coronary heart disease was defined as a previous diagnosis of myocardial infarction (confirmed by characteristic electrocardiographic changes or serial changes in cardiac enzyme activity, or both, or, in the absence of these, a firm clinical diagnosis based on history); stable angina (diagnosed by a history of typical chest pain or discomfort brought on by effort and relieved by rest and/or sublingual nitrates); or revascu- 
Table 2 Characteristics at baseline of the 1906 patients alive and registered with the practice at follow up, by trial group. Values are numbers (percentages) of patients unless stated otherwise

\begin{tabular}{|c|c|c|c|}
\hline & Audit group ( $n=559$ ) & GP recall group $(n=682)$ & Nurse recall group ( $n=665$ ) \\
\hline Mean (SD) age (years) & $66.1(5.4)$ & $66.4(5.6)$ & $65.8(5.8)$ \\
\hline Men & $373(67)$ & $457(67)$ & $469(71)$ \\
\hline \multicolumn{4}{|l|}{ Original diagnosis: } \\
\hline Myocardial infarction (with or without angina) & $294(53)$ & $342(50)$ & $320(48)$ \\
\hline Angina only & $265(47)$ & $340(50)$ & $345(52)$ \\
\hline Mean (SD) No of years since original diagnosis & $7.9(6.1)$ & $8.5(7.7)$ & $8.5(7.1)$ \\
\hline \multicolumn{4}{|l|}{ Complications: } \\
\hline Coronary artery bypass grafting & $92(16)$ & $109(16)$ & $109(16)$ \\
\hline Percutaneous transluminal coronary angioplasty & $43(8)$ & $84(12)$ & $77(12)$ \\
\hline Diabetes & $80(14)$ & $77(11)$ & $66(10)$ \\
\hline Heart failure & $44(8)$ & $65(10)$ & $61(9)$ \\
\hline \multicolumn{4}{|l|}{ Smoking status: } \\
\hline Current smoker & $94(17)$ & $116(17)$ & $126(19)$ \\
\hline Non-smoker & $367(66)$ & $442(65)$ & $424(64)$ \\
\hline Not recorded & $97(17)$ & $122(18)$ & 115 (17) \\
\hline
\end{tabular}

larisation by percutaneous transluminal coronary angioplasty or coronary artery bypass grafting (as recorded in a hospital discharge letter and "operation note" confirming that the procedure(s) took place). Patients who had single episodes of chest pain diagnosed as possible angina but who did not continue to take any antianginal drugs were not included. In all, 2142 patients were identified as having coronary heart disease.

\section{Interventions}

Interventions started in May 1998 and continued until October 1999.

Audit and feedback (audit group)-Practices were given summary audit results at a practice meeting (one practice requested written material only). The results presented were the number of patients with myocardial infarction, angina, and revascularisation; the prevalence of identified coronary heart disease in the practice; and the proportions of patients with "adequate assessment" (see "Study outcomes" for definition) and treatment with antiplatelet drugs, hypotensive agents, and lipid lowering drugs. Anonymised data from other practices in the study were given for comparison. Practices were asked to provide usual care and were given no further support during the trial.

Recall to general practitioner (GP recall group)Practices were given the same patient information as was given to the audit group but were also given the names of patients identified as having coronary heart disease. MM discussed and agreed guidelines for secondary prevention with the practice doctors and gave ongoing support in setting up a register and recall system for regular review of patients with coronary heart disease by their general practitioner.

Recall to nurse clinic (nurse recall group)-Practices were given the same patient information as was given to the GP recall group. The trial's nurse facilitator (LW) gave ongoing support to the practices in setting up a register and recall system for systematic review of patients with coronary heart disease in a nurse led clinic. After discussion and agreement of guidelines for secondary prevention, the practice doctors and nurses agreed the clinic protocol, and the nurses received education to implement it.

\section{Randomisation}

The baseline audit showed considerable variation in the proportion of patients receiving adequate assessment (range $14 \%$ to $38 \%$, mean 29\%), the main outcome at follow up. To achieve baseline balance between groups, blocked random allocation was performed within three strata based on this factor. Randomisation, based on computer generated random numbers, was carried out under observation by a statistician blind to the identity of the practice.

\section{Study outcomes}

The primary outcome was adequate assessment at 18 months' follow up. At the first audit adequate assessment was defined as (a) a record of blood pressure since diagnosis and, if on any occasion this was recorded as exceeding $140 \mathrm{~mm} \mathrm{Hg}$ systolic or $90 \mathrm{~mm}$ $\mathrm{Hg}$ diastolic, a record of a follow up blood pressure in the previous two years; (b) a record of serum cholesterol measurement since diagnosis and, if any reading was $\geqslant 5.5 \mathrm{mmol} / \mathrm{l}$, a record of repeat cholesterol measurement in the previous two years; $(c)$ a record of smoking habit and, for smokers, a record of review in the previous two years. At the second audit the same criteria were applied, except that a blood pressure reading in the previous two years was mandatory. The main secondary outcomes were recorded treatment with hypotensive agents, lipid lowering drugs, and antiplatelet drugs.

Adequate assessment and drug treatment were determined at baseline and follow up from an audit of patients' notes. Of the 2142 patients identified in the first audit as having coronary heart disease, 236 (11\%) had died, moved practice, or were found to have been misdiagnosed before the second audit. Characteristics of the remaining 1906 patients were similar in the three trial groups (table 2).

After the second audit, 1824 patients remained in the trial (a further 9 had died, 30 had moved away, and 43 were thought to be too ill to attend). These were invited to attend their practice for a clinical examination. The invitation letter enclosed a questionnaire including two quality of life instruments (the Dartmouth COOP charts ${ }^{14}$ and the EuroQol questionnaire without the visual analogue scale $\left.{ }^{15}\right) ; 1368(75 \%)$ patients returned the questionnaire and 1342 (74\%) 
Table 3 Patients with coronary heart disease per practice at follow up and percentage of these patients with adequate assessment at baseline and follow up, by trial group. Values are mean (range) percentages unless stated otherwise

\begin{tabular}{|c|c|c|c|c|}
\hline & Audit group ( $n=7)$ & GP recall group $(n=7)$ & Nurse recall group $(n=7)$ & $\begin{array}{l}P \text { value for difference } \\
\text { between groups* }\end{array}$ \\
\hline $\begin{array}{l}\text { Mean No (range) of patients with coronary heart } \\
\text { disease per practice }\end{array}$ & $80(32-140)$ & $97(25-124)$ & $95(26-222)$ & \\
\hline \multicolumn{5}{|l|}{ Adequate assessment (overall): } \\
\hline Baseline & $29(13-49)$ & $31(12-49)$ & $29(17-40)$ & \multirow[t]{2}{*}{$<0.001 \dagger$} \\
\hline Follow up & $52(38-73)$ & $76(54-92)$ & 85 (75-95) & \\
\hline \multicolumn{5}{|l|}{ Blood pressure: } \\
\hline Baseline & $82(68-92)$ & $84(64-95)$ & $85(69-96)$ & \multirow[t]{2}{*}{$<0.001$} \\
\hline Follow up & $86(72-94)$ & $97(96-100)$ & $96(85-98)$ & \\
\hline \multicolumn{5}{|l|}{ Cholesterol: } \\
\hline Baseline & $42(28-64)$ & $48(41-56)$ & $44(30-65)$ & \multirow[t]{2}{*}{0.001} \\
\hline Follow up & $67(46-85)$ & $83(71-95)$ & $88(77-97)$ & \\
\hline \multicolumn{5}{|l|}{ Smoking status: } \\
\hline Baseline & $73(50-91)$ & $71(47-96)$ & $71(46-85)$ & \multirow[t]{2}{*}{0.001} \\
\hline Follow up & $78(56-92)$ & $92(77-100)$ & 95 (88-98) & \\
\hline
\end{tabular}

Mean percentages are weighted by number of patients with coronary heart disease in each practice.

*Based on means adjusted for baseline.

†Nurse recall group $v$ audit group: difference $33 \%$ (95\% confidence interval $19 \%$ to $46 \%$; $\mathrm{P}<0.001)$; GP recall group $v$ audit group: difference $23 \%$ (10\% to $36 \%$; $\mathrm{P}=0.002)$; nurse recall group $v \mathrm{GP}$ recall group: difference $9 \%(-3 \%$ to $22 \% ; \mathrm{P}=0.13)$.

attended (figure). A research nurse who was blind to the patient's allocation group and had no previous involvement with the trial, carried out the clinical assessment. This included blood pressure measurement (two measurements taken five minutes apart with a digital blood pressure monitor) and blood sampling for serum cholesterol and cotinine estimations. All blood analyses were carried out in the same laboratory. A plasma cotinine level of $\leqslant 20 \mathrm{ng} / \mathrm{ml}$ was taken to indicate non-smoking. ${ }^{16}$

\section{Sample size}

We estimated that $25 \%$ of patients would be adequately assessed at baseline $e^{4}$ and that at follow up the proportion adequately assessed would be $35 \%$ in the audit group, $55 \%$ in the GP recall group, and $75 \%$ in the nurse recall group. The sample size calculation was based on detecting the difference between $35 \%$ and $55 \%$. Under individual randomisation 96 patients would be needed in each group for $80 \%$ power $(2 \alpha=0.05)$. To account for the clustered design we applied the cluster inflation factor ${ }^{17}$ and carried out sensitivity analyses assuming an intracluster correlation coefficient between 0.05 and $0.06^{18}$ and a cluster size between 100 and 200. Under any of these assumptions seven practices in each trial arm would give the study a power of at least $82 \%$ (maximum $90 \%$ ), and this trial size was adopted. In practice, the baseline intracluster correlation coefficient for adequate assessment was 0.055 and the mean cluster size 102 (range 28-244). By chance, we recruited exactly 21 practices from Warwickshire; had this not been possible, we would have extended the geographical area of the trial.

\section{Statistical methods}

Analysis followed a prespecified plan. The primary analysis was based on the 21 practice percentages (for dichotomous outcomes) or means (for continuous outcomes). ${ }^{19}{ }^{20}$ The groups were compared by using analysis of variance, weighted by the number of patients in the practice and adjusted for baseline values where available. When significance $(\mathrm{P}<0.05)$ was reached, follow up comparisons between pairs of groups were performed (with no correction for multiple comparisons). We expressed effect size as the difference between groups with a 95\% confidence interval. For dichotomous outcomes, we preferred the measure of risk difference to the odds ratio, which often gave an inflated measure of relative risk.

To incorporate variables at individual level into the models - and for statistical efficiency-analysis based on values for individual patients was also performed, by using hierarchical modelling. ${ }^{21}$ Neither this individual level analysis nor the addition of individual level covariates made any material difference to the results obtained. We have therefore presented only the cluster based results, as we wanted to present risk differences. The analyses were carried out using SPSS (version 9), Stata (version 6), and MLwiN (version 1.10).

\section{Ethical approval}

The Warwickshire regional ethics committee approved the study. In addition, we obtained the consent of the individual general practitioners to audit the case notes of the patients. All patients gave informed consent before attending the clinical assessment.

\section{Results}

\section{Adequate assessment}

At baseline about $30 \%$ of patients were adequately assessed overall; this proportion rose markedly during the trial in all three groups (table 3). The greatest contribution to the overall rise came from the improvement in assessing cholesterol levels, but assessment of smoking status and blood pressure also increased, especially in the GP recall and nurse recall groups.

At follow up the groups differed substantially in the proportions of patients being adequately assessed overall (85\% (566/665) in the nurse recall group; $76 \%$ (521/682) in the GP recall group; and only 52\% (293/ 559 ) in the audit group). After adjustment for baseline levels, the absolute increase in the proportion of patients adequately assessed overall, compared with the audit group, was 33\% (95\% confidence interval $19 \%$ to $46 \%)$ in the nurse recall group and $23 \%(10 \%$ to $36 \%$ ) in the GP recall group. Adequate assessment was higher in the nurse recall group than the GP recall 
Table 4 Mean (range) percentage of patients with coronary heart disease treated with specified drugs at baseline and follow up, by trial group

\begin{tabular}{|c|c|c|c|c|}
\hline & Audit group ( $\mathrm{n}=7$ ) & GP recall group $(n=7)$ & Nurse recall group $(n=7)$ & $\begin{array}{l}\text { P value for difference between } \\
\text { groups* }\end{array}$ \\
\hline \multicolumn{5}{|l|}{ Hypotensive: } \\
\hline Baseline & $67(54-84)$ & $71(56-80)$ & $65(54-71)$ & \multirow[t]{2}{*}{0.35} \\
\hline Follow up & $70(61-83)$ & $73(64-83)$ & $66(58-75)$ & \\
\hline \multicolumn{5}{|c|}{ Lipid lowering: } \\
\hline Baseline & $25(14-37)$ & $24(17-48)$ & $23(17-27)$ & \multirow[t]{2}{*}{0.63} \\
\hline Follow up & $37(9-64)$ & $41(26-68)$ & $40(38-45)$ & \\
\hline \multicolumn{5}{|l|}{ Antiplatelets†: } \\
\hline Baseline & $62(44-77)$ & $73(57-85)$ & $66(48-74)$ & \multirow[t]{2}{*}{$0.017 \ddagger$} \\
\hline Follow up & $74(55-84)$ & $80(68-92)$ & $85(81-89)$ & \\
\hline
\end{tabular}

Mean percentages are weighted by number of patients with coronary heart disease in each practice.

*Based on means adjusted for baseline.

†Among the 1754 patients with no contraindication recorded at baseline or follow up.

fNurse recall group $v$ audit group: difference $10 \%$ (95\% confidence interval $3 \%$ to $17 \% ; P=0.009)$; GP recall group $v$ audit group: difference $2 \%$ ( $-6 \%$ to $10 \%$;

$\mathrm{P}=0.61)$; nurse recall group $v$ GP recall group: difference $8 \%(1 \%$ to $15 \% ; \mathrm{P}=0.031)$.

group, but the difference ( $9 \%$ ( $-3 \%$ to $22 \%))$ was not significant. The three components of adequate assessment all followed a similar pattern.

\section{Drug treatment}

The much higher levels of adequate assessment at follow up in the GP and nurse recall groups were not matched by similar differences in drug treatment (table 4). Prescribing of hypotensive drugs showed minimal change in all three groups, but over two thirds (68\%; $1290 / 1906$ ) of patients were already being treated with hypotensive drugs at baseline. Among the 1782 patients adequately assessed for blood pressure at follow up, raised blood pressure $(>160 \mathrm{~mm} \mathrm{Hg}$ systolic or $>100 \mathrm{~mm} \mathrm{Hg}$ diastolic at the most recent measurement) was observed in $18 \%(84 / 478)$ of patients in the audit group, $17 \%(113 / 663)$ in the GP recall group, and $13 \%(86 / 641)$ in the nurse recall group. Of the 283 patients in all groups with this higher blood pressure, 55 (19\%) were not being treated with hypotensive drugs.

All groups showed an increase in prescribing of lipid lowering drugs, but this increase was no greater in the GP and nurse recall groups than in the audit group. This lack of difference could not be attributed to lower cholesterol levels among the additional patients assessed in the GP and nurse recall groups. At follow up a raised cholesterol level $(>5.5 \mathrm{mmol} / \mathrm{l}$ at the most recent measurement) was recorded in $40 \%(149 / 373)$ of the patients in the audit group, 39\% (221/568) in the GP recall group, and 43\% (251/587) in the nurse recall group, and a cholesterol level of $>6.0 \mathrm{mmol} / \mathrm{l}$ in $24 \%$ (91/373), 22\% (125/568), and 26\% (155/587) of patients respectively. Of the 371 patients in all groups with this higher cholesterol level, 172 (46\%) were not being treated with lipid lowering drugs.

Information about antiplatelet treatment is confined to prescriptions, as we did not have a comprehensive record of self medication with these drugs. Prescribing of antiplatelet drugs was examined only in the 1754 patients with no recorded contraindication at either audit. Prescribing increased in all groups, but at follow up the nurse recall group, with $85 \%$ coverage, had achieved higher levels of prescribing than the GP recall and audit groups. Baseline levels differed considerably across the groups, and after adjustment for these differences the prescribing rates for antiplatelet drugs were $76 \%, 78 \%$, and $86 \%$ in the audit, GP recall, and nurse recall groups respectively. Thus 10\% more patients (95\% confidence interval 3\% to $17 \%$ ) were prescribed antiplatelet drugs in the nurse recall group than in the audit group, and $8 \%$ more patients $(95 \%$ confidence interval $1 \%$ to $15 \%)$ were prescribed antiplatelet drugs in the nurse recall group than in the GP recall group. We did not detect a difference between the GP recall and audit groups (difference $2 \%$ ( $-6 \%$ to $10 \%)$ ).

\section{Clinical examination}

Of the 1824 patients invited for assessment (figure), $1342(74 \%)$ attended (73\% in the audit group, $70 \%$ in the GP recall group, and $78 \%$ in the nurse recall group $(\mathrm{P}=0.41)$. Attendance was higher $(80 \%)$ among patients who were judged adequately assessed at the second audit than among those who were not (64\%) $(\mathrm{P}<0.001$ from hierarchical modelling).

Table 5 shows that mean blood pressure, serum cholesterol (total and high density lipoprotein) level, and smoking status did not differ significantly between the three groups.

\section{Quality of life}

We found no significant or clinically important differences between groups for any dimension of the Dartmouth COOP charts or for EuroQol scores.

\section{Discussion}

We already know from studies of other chronic conditions that high quality care needs to be systematic and that outcome tends to be better when quality assurance is introduced on the basis of registration and planned follow up. ${ }^{22}{ }^{23}$ This trial shows that the simple expedient of setting up a patient register for a general practice markedly increases the probability of planned follow up taking place. It also shows that this benefit can be achieved whether responsibility for follow up lies with the general practitioner or with the practice nurse, and indeed follow up by nurses seems to be more effective. The only significant treatment benefit we observed was an improvement in the prescribing of antiplatelet drugs in the nurse recall group, although in the absence of complete information on self medication we cannot be sure that this reflects a genuine increase of such drugs. As increasing demands are being made on primary care to deliver systematic care for patients 
Table 5 Mean blood pressure and cholesterol, and percentage of coronary heart disease patients assumed not smoking at follow up, by trial group. Values are means (range) unless stated otherwise

\begin{tabular}{|c|c|c|c|c|}
\hline & Audit group ( $n=7$ ) & GP recall group $(n=7)$ & Nurse recall group $(n=7)$ & $\begin{array}{l}\text { P value for difference } \\
\text { between groups }\end{array}$ \\
\hline \multicolumn{5}{|l|}{ Blood pressure $(\mathrm{mm} \mathrm{Hg})$ : } \\
\hline Systolic & $148(136-153)$ & $147(135-153)$ & $148(142-153)$ & 0.82 \\
\hline Diastolic & $81(75-82)$ & $81(75-83)$ & $80(74-87)$ & 0.82 \\
\hline \multicolumn{5}{|l|}{ Cholesterol (mmol/l): } \\
\hline Total & $5.5(5.2-6.1)$ & $5.5(5.0-5.9)$ & $5.4(5.2-5.5)$ & 0.61 \\
\hline High density lipoprotein & $1.2(1.2-1.3)$ & $1.2(1.2-1.3)$ & $1.2(1.1-1.3)$ & 0.83 \\
\hline$\%$ (range) of assumed non-smokers* & $81(69-86)$ & $84(75-88)$ & $83(73-100)$ & 0.58 \\
\hline
\end{tabular}

Means are based on number of patients with coronary heart disease with clinical measurements: blood pressure, $n=1341$; total cholesterol, $n=1286$; high density lipoprotein cholesterol, $n=1264$; cotinine, $n=1271$.

Overall means and mean percentages are weighted by number of patients in each practice with the relevant measurement.

*Plasma cotinine $\leqslant 20 \mathrm{ng} / \mathrm{ml}$.

with chronic disease - without the prospect of a similar increase in the number of general practitioners-this is an important finding.

The trial also showed a lack of difference between the GP and nurse recall groups in clinical outcome. This is entirely consistent with previous trials of secondary prevention of coronary heart disease in general practice that have reported objective measurements of patient risk factors. ${ }^{8} 10$

\section{Limitations of study}

The difficulties of conducting pragmatic intervention trials in primary care are well recognised. ${ }^{24}{ }^{25}$ Although our trial was completed successfully from both a methodological and practical point of view, our results may not be generalisable. The 21 participating practices tended to be large, with good nursing support, and may have been particularly committed to improving their quality of care. Some of the non-participating practices, however, were singlehanded, with minimal nursing support, and it would be difficult for them to implement a nurse recall strategy. Furthermore the observed intervention effect would probably have been greater if the trial had not taken place in the context of a health authority audit initiative relating to patients with coronary heart disease, backed by a financial incentive.

\section{Lack of clinical benefit}

Why are we managing consistently to improve the process of care without achieving any apparent clinical benefit? We suggest several hypotheses, some of which were advanced by the medical and nursing staff in participating practices in informal interviews and discussions after the trial had ended.

Firstly, each intervention was predefined and allocated randomly to practices without assessment of need. To influence clinical outcome it may be important to focus more precisely on the staff training needs and clinical competencies of each practice.

Secondly, room for improvement may be limited in certain areas of care. For example, in our study population previous smoking cessation advice was widespread (over $70 \%$ of patients had their smoking habit recorded in their notes at baseline). Many of the residual smokers must have failed to stop smoking despite this advice in the motivating context of a myocardial infarction. Moreover, even at baseline, over $80 \%$ of patients had had their blood pressure adequately assessed and over two thirds were receiving hypotensive treatment
Thirdly, in the management of raised cholesterol levels, the prescribing of lipid lowering drugs was substantially suboptimal, even after intervention. Several factors may explain this apparent reluctance to initiate the necessary prescribing changes. Not all medical staff were involved in the practice meetings held after randomisation to discuss and agree guidelines for secondary prevention, so commitment to implement change may not have permeated the practice. The impact of prescribing lipid lowering drugs on cash limited prescribing budgets was also mentioned in some practices. Another factor may be a dislike of polypharmacy by both professionals and patients. Minimising the risk of major coronary events often requires five or more drugs in those with diabetes or hypertension, but the need for polypharmacy and sustained behaviour change emphasises the chronic and progressive nature of the disease.

\section{Conclusions}

The lessons to be learned from this trial are threefold: helping practices to set up a practice register of eligible patients increases follow up and adequate assessment; care by nurses is as effective as, and possibly more effective than, systematic care by doctors; and adequate

\section{What is already known on this topic}

Effective preventive care of patients with any chronic disease requires planned and quality assured follow up on the basis of an up to date register

Strategies for changing clinical practice in primary care have been of limited effectiveness

\section{What this study adds}

Setting up a coronary heart disease register for a practice substantially increases follow up and adequate assessment of patients at risk

Improved assessment and follow up does not necessarily improve clinical outcome

Follow up by nurses is as effective as, and may be more effective than, follow up by doctors

Patients are being followed up and adequately assessed without the recommended preventive drugs being prescribed 
assessment does not necessarily translate into better care or clinical outcome. Understanding why this is, and what we should do about it, is the key next step in improving the quality of care of patients with established coronary heart disease.

The ASSIST Trial Collaborative Group also includes G Fowler, E Fullard, K Johnston, A Gray, M Murphy, A Neil, S Thompson, F Wells, Wiles R, and L Youngman.

We thank the patients and staff in each of the participating general practices, including the lead general practitioners (Drs B Bemand, A Boyce, B Carson, J Carter, A Chaudhuri, J Cumming, I Draper, B Gallagher, J Harris, J Hatton, J Hawkins, B Kachhia, S Kavuri, S Purnell-Mallick, C Reily, T Shackley, A Thomas, S Walter, A Watt, J Wilkinson, and M Woo). We also thank the research nurses (L M Ashley-Edden, F J Barker, A Barter, I Bealing, J M Belford, C A Green, J Goodman, H L Hayes, B F Hendrick, J K Kang, T Light, J Maxwell, B Moir-Bussy, R Rae, A A Scanlon, and I Smart) and the seven practice nurses in the nurse recall group who conducted nurse clinics in their respective practices (V Axon, T Colledge, K Collins, R Duffin, R Langman, D Squires, and S Stepney). We thank Tim Lancaster for useful comments on an earlier draft of this manuscript.

Contributors: MM contributed to the study protocol and was medical coordinator of the trial. PY contributed to the study protocol, gave ongoing research leadership, and was responsible for the statistical analysis. LW designed and implemented the nurse led intervention and was nursing coordinator of the trial. RT made an important contribution to the statistical analysis and interpretation. TS initiated and was responsible for the study protocol. DM discussed core ideas and gave intellectual stimulus. MM, PY, TS, and DM drafted the text of the paper with the support of the other authors. TS is guarantor for the study.

Funding: The study was funded by a national research and development programme grant from the NHS Executive. MM received a training fellowship jointly from the Medical Insurance Agency and the Royal College of General Practitioners.

Competing interests: LW has been reimbursed by Pfizer, the manufacturer of atorvastatin, for attending a conference.

1 Shaper AG, Pocock SJ, Walker M, Phillips AN, Whitehead TP, Macfarlane PW. Risk factors for ischaemic heart disease: the prospective phase of the British regional heart study. J Epidemiol Community Health 1985;39:197209.

2 Moher M. Evidence of effectiveness of interventions for the secondary prevention and treatment of coronary heart disease in primary care-a review of the literature. Oxford: NHS Executive, Anglia and Oxford Regional Health Authority, 1995.

3 ASPIRE Steering Group. A British Cardiac Society survey of the potential for the secondary prevention of coronary heart disease: ASPIRE (action on secondary prevention through intervention to reduce events) principal results. Heart 1996;75:334-42.

4 Campbell NC, Thain J, Deans HG, Ritchie LD, Rawles JM. Secondary prevention in coronary heart disease: a baseline survey of provision and possibility in general practice. BMJ 1998;316:1430-4
5 Department of Health. A national service framework for coronary heart disease. London: $\mathrm{DoH}, 2000$.

6 Getting evidence into practice. Effective Health Care 1999;5(1)

7 Campbell N, Thain J, Deans H, Ritchie L, Rawles J, Squair J. Secondary prevention clinics for coronary heart disease: randomised trial of effect on health. BMJ 1998;316:1434-7.

8 Cupples M, McKnight A. Randomised controlled trial of health promotion in general practice for patients at high cardiovascular risk. BMJ 1994;309:993-6.

9 Feder G, Griffiths C, Eldridge S, Spence M. Effect of postal prompts to patients and general practitioners on the quality of primary care after a coronary event (POST): randomised controlled trial. $B M J$ 1999;318:1522-6.

10 Jolly K, Bradley F, Sharp S, Smith H, Thompson S, Kinmonth AL, et al. Randomised controlled trial of follow up care in general practice of patients with myocardial infarction and angina: final results of the Southampton heart integrated care project (SHIP). BMJ 1999;318:706-11.

11 McCartney P, Macdowall W, Thorogood M. A randomised controlled trial of feedback to general practitioners of their prophylactic aspirin prescribing. $B M J$ 1997;315:35-6.

12 Soumerai SB, Avorn J. Principles of educational outreach ("academic detailing") to improve clinical decision making. JAMA 1990;263:549-56.

13 Moher M, Yudkin P, Turner R, Schofield T, Mant D for the ASSIST (Assessment of Implementation Strategies) Trial Collaborative Group. An assessment of morbidity registers for coronary heart disease in primary care. Br J Gen Pract 2000;50:706-9.

14 Nelson E, Wasson J, Kirk J, Keller A, Clark D, Dietrich A, et al. Assessmen of function in routine clinical practice: description of the COOP char method and preliminary findings. J Chronic Dis 1987;40:55-63.

15 EuroQol Group. EuroQol: a new facility for the measurement of health related quality of life. Health Pol 1990;16:199-208.

16 Jarvis MJ, Tunstall-Pedoe H, Feyerabend C, Vesey C, Saloojee Y. Comparison of tests used to distinguish smokers from nonsmokers. Am J Public Health 1987;77:1435-8.

17 Donner A, Birkett N, Buck C. Randomization by cluster: sample size requirements and analysis. Am J Epidemiol 1981;114:906-14

18 Campbell M, Grimshaw J, Steen N. Sample size calculations for cluster randomised trials. J Health Service Pol Res 2000;5:12-6.

19 Donner A, Klar N. Methods for comparing event rates in intervention studies when the unit of allocation is a cluster. Am J Epidemio 1994;140:279-89.

20 Donner A, Klar N. Statistical considerations in the design and analysis of community intervention trials. J Clin Epidemiol 1996;49:435-9.

21 Omar RZ, Thompson SG. Analysis of a cluster randomised trial with binary outcome data using a multilevel model. Stat Med 2000;19:2675-88.

22 Feder G, Griffiths C, Highton C, Eldridge S, Spence M, Southgate L. Do clinical guidelines introduced with practice based education improve care of asthmatic and diabetic patients? A randomised controlled trial in general practices in east London. BMJ 1995;311:1473-8.

23 Pierce M, Lundy S, Palanisamy A, Winning S, King J. Prospective randomised controlled trial of methods of call and recall for cervical cytology screening. BMJ 1989:299:160-2.

24 Wilson S, Delaney BC, Roalfe A, Roberts L, Redman V, Wearn AM, et al. Randomised controlled trials in primary care: case study. BMJ 2000;321:24-7.

25 Rogers S, Humphrey C, Nazareth I, Lister S, Tomlin Z, Haines A. Designing trials of interventions to change professional practice in primary care: lessons from an exploratory study of two change strategies. BMJ 2000;320:1580-3

(Accepted 15 March 2001) 